\title{
Performance Evaluation of Slotted Optical Burst Switching Systems With Quality of Service Differentiation
}

\author{
Onur Ozturk, Ezhan Karasan, Member, IEEE, and Nail Akar, Member, IEEE
}

\begin{abstract}
Slotted optical burst switching (SOBS) has recently caught the attention of the optical networking community due to performance gains achievable with synchronous infrastructures. In this paper, we study the loss probabilities in a slotted optical burst switching node fed with Poisson burst traffic where the fixed burst size is an integer multiple of the slot length. We develop a discrete-time Markov chain (DTMC) based framework to obtain the loss probabilities in systems with and without quality of service (QoS) differentiation. In particular, we focus on analytical modeling of priority scheduling and offset-based QoS differentiation mechanisms for SOBS networks. The latter problem suffers from the curse of dimensionality which we address by a discrete phase type distribution approximation for the discrete Poisson distribution leading to an accurate approximation for the loss probabilities. A hybrid QoS mechanism which jointly utilizes offset-based differentiation together with priority scheduling is also analyzed.
\end{abstract}

Index Terms-Discrete phase type distribution, offset-based differentiation, priority scheduling, QoS, synchronous optical burst switching.

\section{INTRODUCTION}

$\mathbf{O}$ PTICAL burst switching (OBS) was proposed in the late nineties as a candidate transport architecture for the next generation Internet primarily using available optical technologies [1], [2]. The main reason behind the emergence of the OBS paradigm is that the alternative optical circuit switching (OCS), though having widespread acceptance, does not efficiently tap the bandwidth offered by optical transmission systems for bursty Internet Protocol (IP) traffic. Similarly, optical packet switching (OPS) is believed to be (currently) commercially unviable despite its promise of efficient bandwidth use. OBS, on the other hand, combines the advantages of both OCS and OPS and can be viewed as a hybrid and currently viable technology. A recent academic study in [3] shows that OBS might be better suited than OCS for metro-core networks in terms of optical equipment cost. It is pointed out in [4] that

Manuscript received July 03, 2008; revised October 29, 2008 and December 09, 2008. Current version published July 01, 2009. The work described in this paper was carried out with the support of the Building the Future Optical Network in Europe (BONE) project, the Network of Excellence funded by the European Commission through the 7th ICT-Framework Programme, and by the Scientific and Technological Research Council of Turkey (TUBITAK) under the projects 104E047 and 106E046.

The authors are with the Department of Electrical and Electronics Engineering, Bilkent University, Ankara 06800, Turkey (e-mail: ozturk@ee.bilkent. edu.tr; ezhan@ee.bilkent.edu.tr; akar@ee.bilkent.edu.tr).

Digital Object Identifier 10.1109/JLT.2009.2013483
OBS may be implemented in edge networks to reduce the electronic-grooming requirements at the edge-core interface. Another recent study in [5] compares the total cost of ownership (TCO) of a metro Ethernet optical transport network that uses ROADMs (reconfigurable optical add-drop multiplexers) and OBS technology, and the findings of this report show substantial reductions in TCO when using OBS. We believe that more work is still needed to quantify the gain achievable with the use of OBS in different scenarios and for realistic traffic patterns. We note several challenges that need to be resolved for potential widespread acceptance of OBS:

- technological challenges involving lack of optical buffers and efficient wavelength converting equipment,

- lack of OBS standards,

- lack of OBS-based products; there appears to be only one company [6] which has a commercial OBS product.

Keeping these general technical challenges in mind, we focus in this paper on the quality of service (QoS) mechanisms in slotted OBS systems.

In OBS, control and data planes are segregated by means of dedicating one wavelength (or more) for the control plane and the remaining wavelengths for the data plane. In OBS, client packets are aggregated into so-called bursts at the edge of the OBS domain using one of the proposed burst assembly methods [7]. Once a client burst is formed, its reservation request is sent out-of-band on the control channel in the form of a burst control packet (BCP). Under the just enough time (JET) reservation protocol, this BCP consists of the burst length information together with the offset time of the burst [8]. After sending the burst control packet, the ingress edge device waits for the offset time and then sends out the data burst over the data channel, giving the OBS nodes on the path enough time to allocate resources for the upcoming burst. In JET, allocated resources are released as soon as the burst is transmitted by the OBS nodes [1].

Contention in OBS systems arises as a result of two or more incoming bursts contending for the same output wavelength. The most common technique for contention resolution in OBS networks is wavelength conversion [9], [10] although other contention resolution techniques such as fiber delay lines (FDL) [11] or deflection routing can also be used [12]. We assume in the current paper that the OBS system employs only full wavelength conversion for contention resolution where a burst arriving on a certain wavelength can be switched onto any other idle wavelength towards its destination. We do not study relatively limited use of wavelength converters such as partial wavelength conversion or limited range wavelength conversion in this paper [13]. If contention cannot be resolved by wavelength 
conversion, then one or more of the contending bursts will be dropped by the switching system leading to a loss in throughput at higher layers. This is in contrast with electronically packet switched networks in which similar contentions are resolved by electronic buffering leading to low packet loss rates. The reduction of loss probabilities is therefore crucial for the successful deployment of OBS networks for the next generation Internet.

One of the most important implementation decisions that would impact the burst loss probabilities in an OBS network is the mode of operation: asynchronous versus synchronous. Most current OBS proposals are based on asynchronous operation in which bursts have variable sizes and they are not aligned before they enter the optical switch [14]. The node architecture for asynchronous networks is simpler because there is no need for the synchronization stage. However, due to asynchronous operation, there is the possibility of partial overlap between two contending packets destined to the same output fiber. Therefore, asynchronous operation has an adverse effect on system throughput.

Recently, there has been a surge of interest in slotted (or synchronous) optical burst switching (SOBS) systems due to the performance advantages of slotted operation [15]-[17]. SOBS is to OBS, what slotted ALOHA is to ALOHA and similar enhancements in relative performance for SOBS are already shown [17]. In SOBS, data plane is divided into time slots of equal length and data bursts occupy either a single or a multiple number of slots in duration [15] although most existing studies assume that the slot size and the burst length are the same in which case the system is also called SynOBS [16]. The control plane may also be divided into smaller slots in order to accommodate smaller sized control packets or it might use asynchronous operation. In this paper, we focus on slotted operation only in the data plane. To synchronize the arrival times at the OBS switches, the propagation delay of each physical link should be an integer multiple of a time slot which can be achieved by appending to each link an FDL of appropriate length [17]. Several node synchronization issues (that remain to be resolved) are noted for SOBS in regard with small scale fluctuations in the link propagation delays which are kept outside the scope of this paper.

In SOBS, as soon as a burst is assembled at an edge ingress node of an SOBS network, a BCP is sent in the control plane through the network to set up the optical path at each of the core nodes in advance. BCP carries information about the destination address, length, offset time and the carrier wavelength of the burst to the core nodes. Then in the closest data slot after the required basic offset time (for header processing at the intermediate nodes), the burst transmission is initiated. Although bursts are allowed in SOBS to vary in size depending on the assembly algorithm, we assume in this paper that the burst length is a fixed integer multiple $(L \geq 1)$ of the slot length. The advantages of fixed burst sizes have been reported in [18]. An SOBS implementation with $L>1$ is a middle ground alternative between asynchronous OBS with fixed burst sizes (the burst size is fixed but the slot length approaches to zero or equivalently $L \rightarrow \infty$ ) and SynOBS $(L=1)$ and yields the following advantages:

- The major cost of slotted operation in SOBS is the need for additional FDLs appended to each link [17]. However if the slot length is chosen to be small relative to the burst size, i.e., $L>1$, the required FDL lengths will then be shorter as well, reducing FDL costs.

- When a burst is formed, the burst needs to wait until the next data slot. If the slot length is large, then such delays will be longer. On the other hand, with $L>1$, it is possible to reduce such delays stemming from slotted operation.

- SOBS node needs to make scheduling decisions for all bursts coming into the OBS node in a given slot. Since the time required to run a scheduling algorithm is rather limited, it might be appropriate to reduce the number of bursts for which a scheduling decision is to be made. A reduction factor of $L$ in the number of items to be scheduled is possible for SOBS relative to SynOBS.

One of the critical issues in OBS networks is to provide quality of service among data bursts that belong to different service classes. In electronically packet switched networks, QoS is provided by means of per-class queueing, buffer management, and advanced scheduling mechanisms. However, lack of buffering limits the number of options for QoS provisioning in OBS networks. The most popular QoS technique is the offset-based technique in which high priority (HP) bursts are assigned longer offset times than low priority (LP) bursts [19]. For SOBS, the offset-based QoS technique reduces to sending high priority data bursts not right at the next data slot after the arrival of this BCP, but at the following time slot, which we call the unity-offset QoS scheme. In case larger delays are tolerable for high priority traffic, one can optionally send the high priority data burst $M>1$ data slots after the closest data slot. This scheme is called the non-unity-offset QoS scheme.

Another option for providing QoS in SOBS is that, since the data bursts arrive at the same time, all BCPs corresponding to the bursts to arrive at a particular data slot are processed together using a scheduling algorithm providing preferential treatment to HP bursts. This mechanism is called priority scheduling in this paper and we note that one does not need to use offset-based differentiation in case $L=1$ for a system with priority scheduling. When $L>1$, priority scheduling and offset schemes can work in conjunction for improved preferential treatment for HP traffic. We also limit the number of traffic classes to two in this study although most of the results are extensible to more than two traffic classes.

In this paper, we propose a discrete-time Markov chain (DTMC) based performance evaluation framework for slotted OBS systems when the burst length is a fixed integer multiple, $L \geq 1$, of the data slot size. Motivated by Poisson arrival assumptions used commonly for asynchronous OBS systems, we assume that the number of reservation requests (for HP and LP traffic) arriving within a data slot and destined to a tagged fiber with $W$ wavelengths is Poisson distributed. We also assume that arrivals within non-overlapping slots are independent. With these assumptions in place, we analytically find the burst loss probabilities when we have i) best-effort SOBS (no QoS differentiation); ii) QoS with priority scheduling; iii) QoS with unity-offset; iv) QoS with both priority scheduling and unity-offset; v) QoS with priority scheduling and non-unity offset. Scenarios i), ii), and iv) are exactly solved in this paper, whereas an exact solution for iii) is presented but when the Poisson distribution is approximated by a discrete phase type distribution to cope with exploding state spaces. Scenario v) 
is tackled using linear regression due to the curse of dimensionality problem arising for this particular scenario. We also note that the analytical methods proposed in this paper can also be used to approximate the loss probabilities for asynchronous OBS with fixed burst sizes as the parameter $L \rightarrow \infty$.

Analysis of most queueing networks relies on the solution of individual nodes and using fixed point iterations when a product form solution for the path loss probability does not exist. Fixed point iterations have been recently used for the performance analysis of asynchronous OBS networks [20], [21]. Although the analysis of slotted OBS systems presented in this paper involves just a single node, it can be viewed as a first step towards the analysis of end-to-end loss probabilities in slotted OBS networks.

The remainder of the paper is organized as follows. In Section II, we describe our DTMC based framework for the case of best-effort SOBS and study the effect of $L$ on burst loss probabilities in order to assess the performance gains of SOBS relative to OBS. We extend this framework to the analysis of QoS with priority scheduling in Section III. In Section IV, we analyze the scenario when both priority scheduling and unity-offset-based QoS differentiation is employed. The case of using only unity-offset-based differentiation is analyzed in Section V. We provide our numerical results in Section VI to validate the effectiveness of the proposed framework. In this section, we also introduce a numerical method based on regression in order to approximate the case of non-unity offsets. We conclude in the final section.

\section{BEST-EFFORT SOBS}

We consider an SOBS node with $W$ wavelength channels per output fiber with full wavelength conversion capability. We also focus on the performance of a single tagged output fiber. In besteffort SOBS, there is no QoS differentiation and for a given slot, bursts destined for the tagged fiber arrive according to a Poisson distribution with mean $\lambda$. The burst arrival process is also assumed to be independent for two different data slots. The probability mass function (PMF) for the number of burst arrivals in a given data slot is then given by

$$
q_{(i)}=\frac{e^{-\lambda} \lambda^{i}}{i !}, \quad i \geq 0
$$

where $q_{(i)}$ denotes the probability of $i$ burst arrivals in a given slot. The associated cumulative distribution function (CDF) and the complementary CDF (CCDF) denoted by $F_{(i)}$ and $Q_{(i)}$, respectively, are given by

$$
\begin{aligned}
F_{(i)} & =\sum_{k=0}^{i} q_{(k)}, \\
Q_{(i)} & =\sum_{k=i+1}^{\infty} q_{(k)} .
\end{aligned}
$$

We assume that the processing delay of the $\mathrm{BCP}$ at each node is negligible compared to the duration of a data slot. Meanwhile, the data burst sizes are fixed to $L$ slots. The load on the tagged fiber is then written as

$$
\rho=\frac{\lambda L}{W}
$$

For best-effort SOBS, we observe the tagged fiber at the end of data slots. We assume that arrivals take place just at the beginning of data slots. The variable $s^{i}$ denotes the number of wavelength channels that are already reserved for $i$ more slots starting from the end of a data slot, $0 \leq i \leq L-1$, at the observation instants. The best-effort SOBS system is described by a DTMC having the state space, $\mathbf{S}$, given by

$$
\mathbf{S}=\left\{\mathbf{s}=\left(s^{0}, s^{1}, \ldots, s^{L-2}, s^{L-1}\right): s^{i} \geq 0, \sum_{i=0}^{L-1} s^{i}=W\right\} \text {. }
$$

For illustration purposes, the operation for the SOBS system is given in Fig. 1 for a scenario with $W=3$ and $L=2$. In this example, we have a two-dimensional state space $\mathbf{S}=\{(3,0),(2,1),(1,2),(0,3)\}$. As an example, just before the time epoch 0 , there are no bursts scheduled in the system for $t \geq 0$, therefore the system state is $(3,0)$ at $t=0^{-}$. At $t=0$, two new bursts arrive and they are both scheduled for the next two data slots. Therefore, the new system state becomes $(1,2)$ at $t=1^{-}$. We also provide in Fig. 2 the transition probabilities of the Markov chain for this particular example in terms of $q_{(i)}$ and $Q_{(i)}, i \geq 0$.

It is well-known that the cardinality of the $L$-dimensional state space $\mathbf{S}$ is $N=\left(\begin{array}{c}W+L-1 \\ W\end{array}\right)$ which is quite manageable when compared with the brute force approach of taking the cardinality as $L^{W}$ [22]. This reduction in the state space dimensionality stems from the full wavelength conversion assumption. As an example, for $W=10$ and $L=5, N=\left(\begin{array}{l}14 \\ 10\end{array}\right)=1001$, which is far smaller than $5^{10}$.

The transition probabilities of the general Markov chain for general $L$ and $W$ are given in Table I. We note that the resulting Markov chain is irreducible and aperiodic for any choice of $L$ and $W$ and it therefore has a unique steady state distribution. The notation $\pi(\mathbf{s})$ is used to denote the steady state probability that the Markov chain is visiting state $\mathbf{s} \in \mathbf{S}$. The burst loss probability for best-effort SOBS is then given by

$$
P_{\text {loss }}=\frac{1}{\lambda} \sum_{\mathbf{s} \in \mathbf{S}} \pi(\mathbf{s}) \mu(\mathbf{s})
$$

where $\mu(\mathbf{s})$ is the expected number of burst drops for a given state $\mathbf{s}=\left(s^{0}, s^{1}, \ldots, s^{L-2}, s^{L-1}\right)$ and is given by

$$
\begin{aligned}
\mu(\mathbf{s}) & =\sum_{i=s^{0}+1}^{\infty} q_{(i)}\left(i-s^{0}\right) \\
& =\lambda Q_{\left(s^{0}-1\right)}-s^{0} Q_{\left(s^{0}\right)} .
\end{aligned}
$$

We now study the system when we vary the parameter $L$. For this purpose, we fix the burst size but change the size of the data slot in order to vary $L$ while controlling the system load $\rho$ by means of varying $\lambda$. With this, the best-effort SOBS system approaches to an $M / D / c / c$ system as $L \rightarrow \infty$ when $\rho$ is fixed with $c=W$ servers and deterministic service times. Since the loss probabilities for an $M / G / c / c$ system are insensitive to the service time distribution, the loss probability is expressed using the Erlang-B formula developed for the $M / M / c / c$ queue as

$$
P_{\text {loss }}=\frac{\rho^{W} / W !}{\sum_{i=0}^{W} \rho^{i} / i !} \text { as } L \rightarrow \infty .
$$




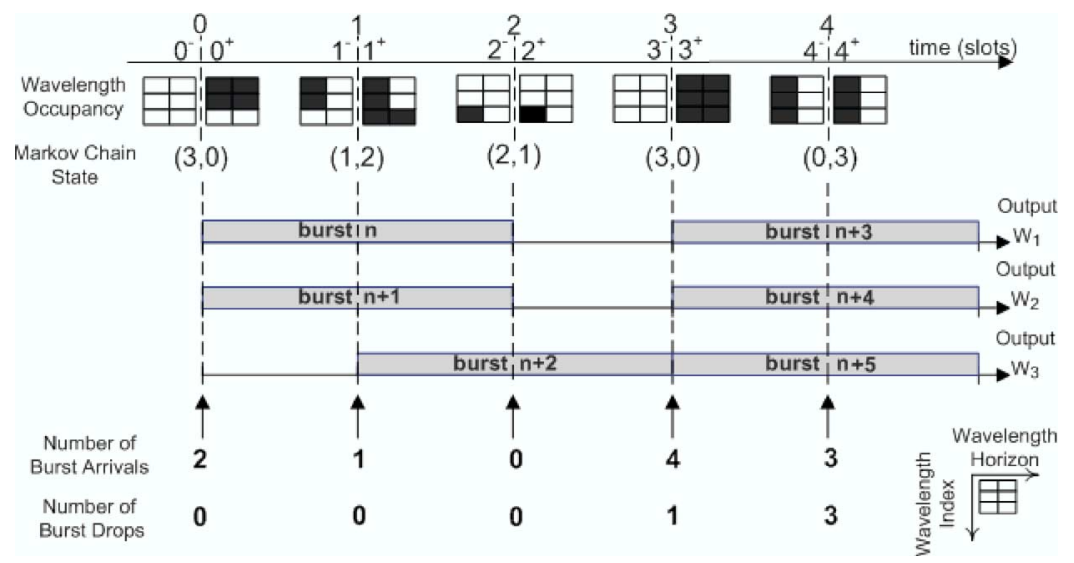

Fig. 1. Illustration of the evolution of the DTMC for the scenario $W=3$ and $L=2$.

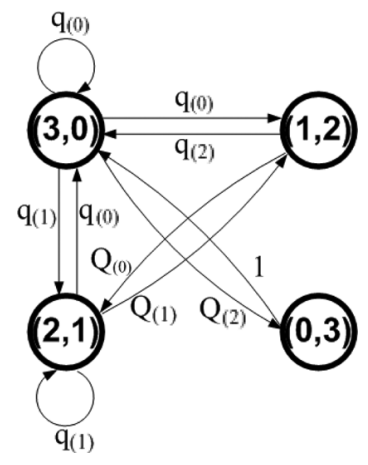

Fig. 2. Example DTMC with $W=3$ and $L=2$.

TABLE I

State Transitions From a Given State $\mathbf{s}=\left(s^{0}, s^{1}, \ldots, s^{L-2}, s^{L-1}\right)$ OF THE DTMC FOR BEST-EFFORT SOBS

\begin{tabular}{|l|l|l|}
\hline \multicolumn{3}{|c|}{ Current State: $\mathbf{s}=\left(s^{0}, s^{1}, \ldots, s^{L-2}, s^{L-1}\right)$} \\
\hline Next State: $\mathbf{s}=$ & Condition & Probability \\
\hline$\left(s^{0}+s^{1}-i, s^{2}, \ldots, s^{L-1}, i\right)$ & $i<s^{0}$ & $q_{(i)}$ \\
\hline$\left(s^{1}, s^{2}, \ldots, s^{L-1}, s^{0}\right)$ & $\forall i \geq s^{0}$ & $Q_{\left(s^{0}-1\right)}$ \\
\hline
\end{tabular}

For SynOBS where $L=1$, the Markov chain has a single state in which all wavelength channels are available. In this special case, the loss probability is simply written as

$$
P_{\text {loss }}=\frac{1}{\lambda}\left(\lambda Q_{(W-1)}-W Q_{(W)}\right) .
$$

The loss probabilities are computed by using the DTMC model presented in this section for $W=2$ and 8 , and for varying $\rho$ and $L$. The results are plotted in Fig. 3(a) and (b). We compare these results to those obtained using the Erlang-B formula for the case of $L \rightarrow \infty$. It is clear that the SOBS results converge to these of the $M / M / c / c$ system as $L \rightarrow \infty$, i.e., as the slot length becomes infinitely small. With a proper choice of $L$, e.g., $L=8$, we show that it is also possible to approximate asynchronous OBS systems using the approach of this paper.

In Fig. 4(a) and (b), the loss probabilities are plotted as a function of $L$, where the dotted lines are obtained using the Erlang-B formula, i.e., for $L \rightarrow \infty$. Our numerical results demonstrate that the convergence of the loss probabilities as the parameter $L$ increases is rather rapid for higher loads and lower number of wavelengths. Consequently, performance gain of synchronizing the OBS network is more prominent for lower loss rates. It is observed from Fig. 4(a) that an SOBS configuration of $W=6$ and $L=2$ yields the same $P_{\text {loss }}$ with that of the asynchronous OBS having $W=8$. A similar observation can be made in Fig. 4(b), as an SOBS node provides with a lower $P_{\text {loss }}$ as compared to an asynchronous OBS. It is readily seen from Fig. 4(b) that an SOBS node can handle larger amount of traffic, i.e., higher $\rho$, than an asynchronous OBS node while providing the same $P_{\text {loss }}$.

\section{SOBS With PRIORITY SCHEDULING QOS MECHANISM}

In this section, the DTMC-based analysis of best-effort SOBS is extended to QoS with priority scheduling. We assume that the high priority (HP) and low priority (LP) burst arrivals destined to the tagged fiber are Poisson distributed with parameters $\lambda_{h}$ and $\lambda_{l}$, respectively. The parameter $\lambda=\lambda_{h}+\lambda_{l}$ denotes the mean number of burst arrivals in a given slot. Let $q_{(i)}^{h}\left(q_{(i)}^{l}\right)$, $F_{(i)}^{h}\left(F_{(i)}^{l}\right)$, and $Q_{(i)}^{h}\left(Q_{(i)}^{l}\right)$ denote the PMF, CDF, and the CCDF for the HP (LP) arrivals in a given slot. Also let $P_{\text {loss }}^{h}$ and $P_{\text {loss }}^{l}$ denote the loss probabilities seen by HP and LP traffic, respectively. In priority scheduling mode of operation, the SOBS node first allocates resources for HP bursts and the remaining resources, if any, are then used for LP bursts. It can be observed that the DTMC model corresponding to the best-effort SOBS case, which was presented in Section II, can be directly used also for the priority scheduling case. However, we need to take priority scheduling into consideration while calculating the loss probabilities from the resulting steady state probabilities of the DTMC. The loss probability for HP bursts is given by

$$
P_{\mathrm{loss}}^{h}=\frac{1}{\lambda^{h}} \sum_{\mathbf{s} \in \mathbf{S}} \pi(\mathbf{s}) \mu^{h}(\mathbf{s})
$$

where

$$
\begin{aligned}
\mu^{h}(\mathbf{s}) & =\sum_{i=s^{0}+1}^{\infty} q_{(i)}^{h}\left(i-s^{0}\right), \\
& =\lambda_{h} Q_{\left(s^{0}-1\right)}^{h}-s^{0} Q_{\left(s^{0}\right)}^{h}
\end{aligned}
$$

and $\mathbf{s}=\left(s^{0}, s^{1}, \ldots, s^{L-2}, s^{L-1}\right)$. 


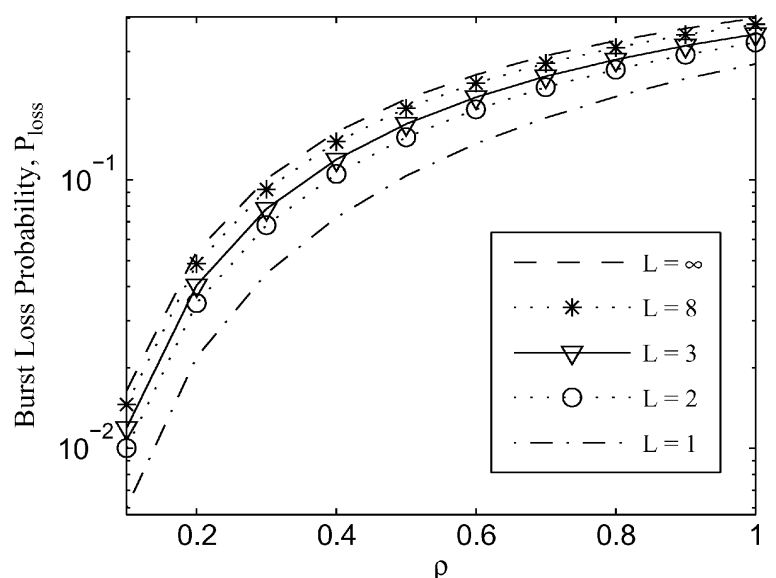

(a)

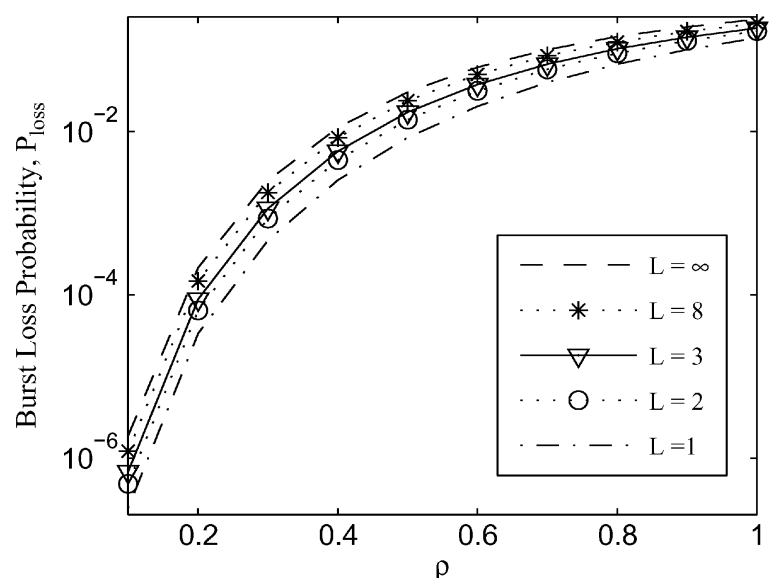

(b)

Fig. 3. Loss probabilities for a best-effort SOBS system for varying $\rho$ and for different values of $L$. (a) $W=2$. (b) $W=8$.

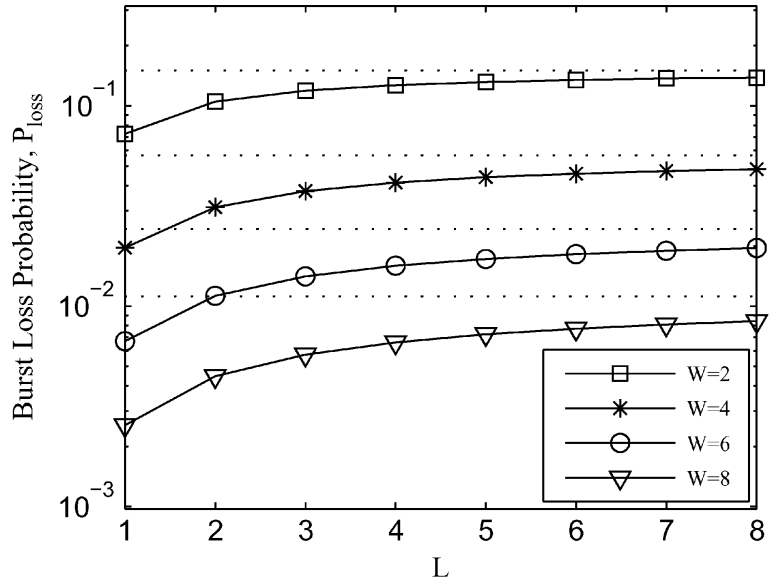

(a)

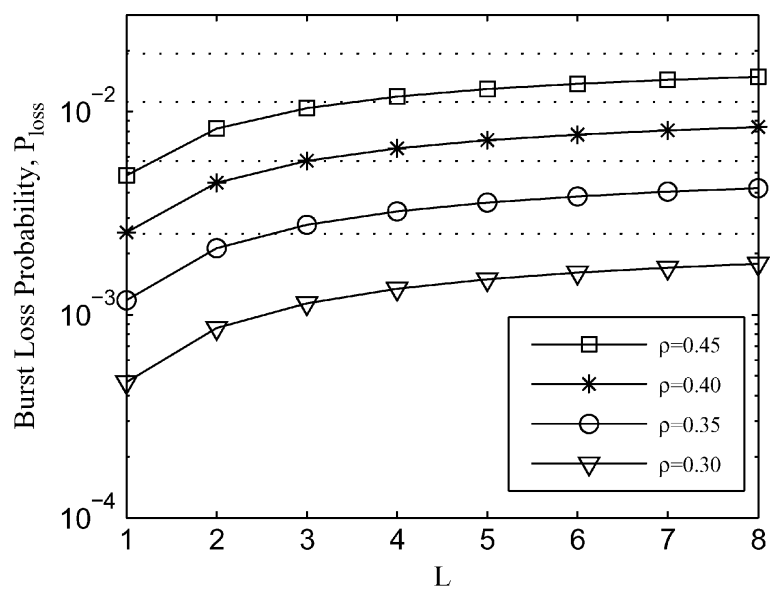

(b)

Fig. 4. Loss probabilities for a best-effort SOBS system for varying $L$ and for different values of $W$. (a) $\rho=0.4$. (b) $W=8$.

The form of (2) is the same as (1) since HP bursts experience the full availability of the resources as in the case of best-effort SOBS. However, the expression for $P_{\text {loss }}^{l}$ is slightly different:

$$
P_{\text {loss }}^{l}=\frac{1}{\lambda^{l}} \sum_{\mathbf{s} \in \mathbf{S}} \pi(\mathbf{s}) \mu^{l}(\mathbf{s})
$$

where

$$
\begin{aligned}
\mu^{l}(\mathbf{s})= & \sum_{k=0}^{s^{0}-1} q_{(k)}^{h} \sum_{i=s^{0}-k+1}^{\infty} q_{(i)}^{l}\left[i-\left(s^{0}-k\right)\right]+\sum_{k=s^{0}}^{\infty} q_{(k)}^{h} \lambda_{l}, \\
= & \sum_{k=0}^{s^{0}-1} q_{(k)}^{h}\left[\lambda_{l} Q_{\left(s^{0}-k-1\right)}^{l}-\left(s^{0}-k\right) Q_{\left(s^{0}-k\right)}^{l}\right] \\
& +\lambda_{l} Q_{\left(s^{0}-1\right)}^{h} .
\end{aligned}
$$

\section{SOBS WITH PRIORITY SCHEDULING AND UNITY OFFSET: A HYBRID QOS MECHANISM}

We now study a scheme where priority scheduling is applied together with the unity offset scheme, i.e., $M=1$, for HP traffic. In this hybrid scheme, HP bursts are given the advantage of making a reservation one data slot in advance. Therefore, when a BCP for an HP burst arrives, the corresponding burst is not to be scheduled for the first data slot at that moment, but instead for the next available data slot in time. On the other hand, when a BCP for an LP burst arrives, its corresponding burst is scheduled for the first data slot. In this scheme, all BCPs arriving within the same slot are processed together but LP bursts can be scheduled only after all HP bursts are scheduled. This scheme is described by a DTMC with state space $\mathbf{S}$ :

$$
\mathbf{S}=\left\{\mathrm{s}=\left(s^{0}, s^{1}, \ldots, s^{L-1}, s^{L}\right): s^{i} \geq 0, \sum_{i=0}^{L} s^{i}=W\right\}
$$

where $s^{i}$ is the number of wavelengths that are reserved for $i$ more slots. In particular, $s^{L}$ denotes the number of HP bursts scheduled in the previous slot. The cardinality of the $L+1$ dimensional state space $\mathbf{S}$ is then $N=\left(\begin{array}{c}W+L \\ W\end{array}\right)$. The description of the Markov chain together with the state transition probabilities are presented in Table II in which the variables $i$ and $j$ denote the number of HP and LP bursts arriving in the same data slot, respectively. 
TABLE II

State Transitions From a Given State $\mathbf{s}=\left(s^{0}, s^{1}, \ldots, s^{L-1}, s^{L}\right) \in \mathbf{S}$ FOR SOBS With QoS Differentiation USING BOTH PRIORITY SCHEDULING AND UNITY-OFFSET

\begin{tabular}{|l|l|l|}
\hline \multicolumn{3}{|c|}{ Current State: $\mathbf{s}=\left(s^{0}, s^{1}, \ldots, s^{L-1}, s^{L}\right)$} \\
\hline \multicolumn{1}{|c|}{ Next State: $\mathbf{s}=$} & \multicolumn{1}{|c|}{ Condition } & \multicolumn{1}{c|}{ Probability } \\
\hline$\left(s^{0}+s^{1}-i-j, s^{2}, \ldots, s^{L}+j, i\right)$ & $i \leq s^{1}, j<s^{0}$ & $q_{(i)}^{h} q_{(j)}^{l}$ \\
\hline$\left(s^{1}-i, s^{2}, \ldots, s^{L}+s^{0}, i\right)$ & $i \leq s^{1}, \forall j \geq s^{0}$ & $q_{(i)}^{h} Q_{\left(s^{0}-1\right)}^{l}$ \\
\hline$\left(s^{0}+s^{1}-i-j, s^{2}, \ldots, s^{L}+j, i\right)$ & $s^{1}<i<s^{0}+s^{1}, j<s^{0}+s^{1}-i$ & $q_{(i)}^{h} q_{(j)}^{l}$ \\
\hline$\left(0, s^{2}, \ldots, s^{L}+s^{0}-\left(i-s^{1}\right), i\right)$ & $s^{1}<i<s^{0}+s^{1}, \forall j \geq s^{0}+s^{1}-i$ & $q_{(i)}^{h} Q_{\left(s^{0}+s^{1}-i-1\right)}^{l}$ \\
\hline$\left(0, s^{2}, \ldots, s^{L}, s^{1}+s^{0}\right)$ & $\forall i \geq s^{0}+s^{1}$ & $Q_{\left(s^{0}+s^{1}-1\right)}^{h}$ \\
\hline
\end{tabular}

The loss probabilities for this system are derived next. The loss probability for HP bursts is given by

$$
P_{\mathrm{loss}}^{h}=\frac{1}{\lambda_{h}} \sum_{\mathbf{s} \in \mathbf{S}} \pi(\mathbf{s}) \mu^{h}(\mathbf{s})
$$

where

$$
\begin{aligned}
\mu^{h}(\mathbf{s}) & =\sum_{i=s^{0}+s^{1}+1}^{\infty} q_{(i)}^{h}\left(i-\left(s^{0}+s^{1}\right)\right) \\
& =\lambda_{h} Q_{\left(s^{0}+s^{1}-1\right)}^{h}-\left(s^{0}+s^{1}\right) Q_{\left(s^{0}+s^{1}\right)}^{h}
\end{aligned}
$$

whereas the loss probability for LP bursts is given by

$$
P_{\text {loss }}^{l}=\frac{1}{\lambda_{l}} \sum_{\mathbf{s} \in \mathbf{S}} \pi(\mathbf{s}) \mu^{l}(\mathbf{s})
$$

where

$$
\begin{aligned}
\mu^{l}(\mathbf{s})= & \sum_{k=0}^{s^{1}} q_{(k)}^{h} \sum_{i=s^{0}+1}^{\infty} q_{(i)}^{l}\left(i-s^{0}\right) \\
& +\sum_{k=s^{1}+1}^{s^{0}+s^{1}-1} q_{(k)}^{h} \sum_{i=s^{0}+s^{1}-k+1}^{\infty} q_{(i)}^{l}\left[i-\left(s^{0}+s^{1}-k\right)\right] \\
& +\sum_{k=s^{0}+s^{1}}^{\infty} q_{(k)}^{h} \sum_{i=0}^{\infty} q_{(i)}^{l} i \\
= & F_{\left(s^{1}\right)}^{h}\left[\lambda_{l} Q_{\left(s^{0}-1\right)}^{l}-s^{0} Q_{\left(s^{0}\right)}^{l}\right] \\
& +\sum_{k=s^{1}+1}^{s^{0}+s^{1}-1} q_{(k)}^{h}\left[\lambda_{l} Q_{\left(s^{0}+s^{1}-k-1\right)}^{l}\right. \\
& +\lambda_{l} Q_{\left(s^{0}+s^{1}-1\right)}^{h} .
\end{aligned}
$$

\section{SOBS With Unity-OFFSET QOS MECHANISM}

In this QoS scheme, a scheduling decision is made immediately for a BCP upon its arrival as opposed to the priority scheduling where all BCPs arriving within the same slot are processed together. Similar to the previous section, when a BCP for an LP (HP) burst arrives, its corresponding burst is scheduled for the first (second) data slot occurring after the instant of BCP arrival.
Typically, the control plane is asynchronous and BCPs do not arrive at once and they will be processed one by one. For this scenario, an option is to use a continuous-time stochastic model (e.g., Poisson arrivals) for control plane traffic in conjunction with a discrete-time traffic model for the data plane. However, hybrid models of this nature are hard to solve analytically. Instead, we propose a discrete phase-type distribution (D-PH) as an approximation to the discrete Poisson distribution which can then be used for modeling sequential processing of BCPs as in the case of asynchronous OBS.

We first start with the definition of a D-PH distribution. Assume that we have a DTMC with $m$ transient states and one absorbing state with a probability transition matrix of the form

$$
R=\left[\begin{array}{cc}
1 & 0 \\
h & T
\end{array}\right] \text {. }
$$

Clearly, the states $k, 1 \leq k \leq m$, are transient and the state 0 is absorbing. We also have $T e+h=e$ with $e$ being a column vector of ones of appropriate size. Let the initial probability row vector be partitioned $(d, v)$ for a scalar $d$ and a row vector $v$ of size $m$. The distribution of the first time to the absorbing state is called a discrete phase type distribution characterized with the pair $(v, T)$ and the transient states are called phases. Most existing studies assume that $d=0$ in which case the distribution does not have a probability mass at zero. However, in this study we use the more general D-PH distributions with nonzero $d$. Let $x$ be the random variable whose distribution is D-PH type. The PMF of this distribution (denoted by $q_{(i)}^{x}$ ) is then given by

$$
q_{(i)}^{x}= \begin{cases}v T^{i-1} h, & i \geq 1 \\ d, & i=0\end{cases}
$$

The factorial moments of a D-PH distributed random variable $x$ can be found through the following expression:

$$
E[x(x-1) \cdots(x-k+1)]=k ! v(I-T)^{-k} T^{k-1} e .
$$

A D-PH type renewal process is a sequence of iid random variables each of which is D-PH distributed.

In the unity offset scheme, we envision a one by one processing of corresponding BCPs. Therefore, we propose to use a D-PH type renewal process to approximate discrete Poisson distributed BCP arrivals. A discrete phase type distribution with a single phase is very limited in its use as an effective approximation. Therefore we propose to use a distribution with two 


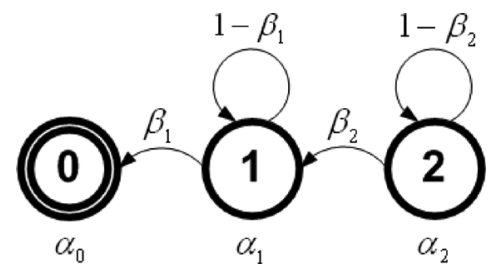

Fig. 5. Markov chain for 2-phase acyclic D-PH type distribution.

phases. The underlying discrete phase type distribution we propose is based on the Markov chain with three states 0, 1, and 2, given in Fig. 5 and characterized with

$$
\begin{aligned}
v & =\left[\begin{array}{ll}
\alpha_{1} & \alpha_{2}
\end{array}\right] \\
T & =\left[\begin{array}{cc}
1-\beta_{1} & 0 \\
\beta_{2} & 1-\beta_{2}
\end{array}\right] .
\end{aligned}
$$

State 0 is the absorbing state and the other two states are transient. At the beginning of a slot, the Markov chain is in state $i$, $i=0,1,2$, with probability $\alpha_{i}$. In state 0 , there is no BCP arrival, and in states 1 and 2, there is a single BCP arrival. Once the absorbing state is reached, it means the end of the current slot and a procedure for the next slot is initiated again by starting at state $i$ with probability $\alpha_{i}$. The transition probabilities of the Markov chain are found so that the first two moments of the D-PH distribution are the same as that of the discrete Poisson distribution. The details of the parameter matching procedure are given in Appendix I. D-PH parameter matching is a well-known problem of applied probability; see for example [23]. However, D-PH distributions dealt with in [23] do not have a probability mass at the origin and in our algorithm, we need to take into consideration nonzero probability masses at the origin. Thus, we introduce $d=\alpha_{0}$ corresponding to the case where no bursts arrive at the system. Note that D-PH type distributions are mostly used in the literature to model discrete time between certain events such as packet/burst arrivals. However, we interpret the state transitions of the D-PH type Markov chain as burst arrivals within a slot rather than the elapsed time between two consecutive burst arrivals.

Given the approximative model for BCP arrivals, we have a new Markov chain with state space:

$$
\begin{aligned}
& \mathbf{S}=\left\{\mathbf{s}=\left(s^{0}, s^{1}, \ldots, s^{L-1}, s^{L}, s^{L+1}, P\right)\right. \\
&\left.s^{i} \geq 0, \sum_{i=0}^{L+1} s^{i}=W, P=0,1,2\right\} .
\end{aligned}
$$

In $\mathbf{S}$, probability of an arrival event depends solely on the last sub-state $P$. Bernoulli trials with probabilities $p_{h}$ and $p_{l}$ are used to generate the HP and LP traffic respectively from the arrival realizations. They are defined as

$$
\begin{aligned}
p_{h} & :=\operatorname{Pr}\{\text { an arriving burst is HP }\}=\frac{\lambda_{h}}{\lambda_{h}+\lambda_{l}} \\
p_{l} & :=\operatorname{Pr}\{\text { an arriving burst is LP }\}=\frac{\lambda_{l}}{\lambda_{h}+\lambda_{l}}
\end{aligned}
$$

In offset-based QoS, maximum observable horizon by the incoming bursts extends to $s^{L+1}$ which is explained below together with the applied scheduling policy.

- HP bursts are assumed to arrive one slot after their control packets. Hence, $\mathbf{S}$ evolves trying to reserve one of the $s^{1}$ wavelengths first and otherwise, i.e., if $s^{1}=0$, one of the $s^{0}$ wavelengths. If both states are vacant, the burst is lost. Otherwise, $s^{L+1}$ is incremented by one. The notation $s^{L+1}$ corresponds to the sub-state temporarily holding HP bursts until a transition to the absorbing state, i.e., $P=0$, occurs. This transition represents the end of arrivals for that slot.

- LP bursts are assumed to arrive synchronized with their control packets. Hence, $\mathbf{S}$ evolves trying to reserve one of the $s^{0}$ wavelengths. If $s^{0}=0$, the LP burst is lost. Otherwise $s^{L}$ is incremented by one. The term $s^{L}$ corresponds to both the HP bursts whose BCPs were received one slot before and currently scheduled LP bursts until the absorbtion time.

- After a transition to the absorbing state, $P=0$, every state $s^{i}$ transfers its content to the previous state $s^{i-1}$ which means advancing in time. By this evolution, $s^{L+1}$ becomes 0 and $s^{0}$ becomes $s^{0}+s^{1}$.

Based on this scheduling policy, state transitions are explicitly given in Tables III, IV, and V. The cardinality of the $(L+2)$ dimensional state space $\mathbf{S}$ is given by

$$
N=\left(\begin{array}{c}
W+L \\
W
\end{array}\right)+\left(\begin{array}{c}
W+L+1 \\
W
\end{array}\right)+\left(\begin{array}{c}
W+L+1 \\
W
\end{array}\right) .
$$

The summed terms correspond to the states in which $P=0,1$, and 2, respectively. As $P=0$ depicts that no burst has arrived at the optical node from the beginning of a slot, $s^{L+1}$ holding the number of HP bursts arriving in that slot must be zero explaining the lower upper limit for the first term. The burst loss probabilities of HP and LP classes are found as follows. We first define

$$
\begin{aligned}
e(\mathbf{s}) & :=\operatorname{Pr}\{\text { an arrival occurs in state } \mathbf{s}\} \\
& = \begin{cases}1-\alpha_{0} & \text { if } P=0 \\
1-\beta_{1} & \text { if } P=1 \\
1 & \text { if } P=2\end{cases} \\
\gamma^{h}(\mathbf{s}) & :=\operatorname{Pr}\{\text { an arriving HP burst is blocked in state } \mathbf{s}\} \\
& = \begin{cases}1 & \text { if } s^{0}+s^{1}=0 \\
0 & \text { otherwise }\end{cases} \\
\gamma^{l}(\mathbf{s}) & :=\operatorname{Pr}\{\text { an arriving LP burst is blocked in state } \mathbf{s}\} \\
& = \begin{cases}1 & \text { if } s^{0}=0, \\
0 & \text { otherwise }\end{cases}
\end{aligned}
$$

Loss probabilities for HP and LP bursts are then given by

$$
\begin{aligned}
& P_{\text {loss }}^{h}=\frac{\sum_{\mathbf{s} \in \mathbf{S}} \pi(\mathbf{s}) \gamma^{h}(\mathbf{s}) e(\mathbf{s}) p_{h}}{\sum_{\mathbf{s} \in \mathbf{S}} \pi(\mathbf{s}) e(\mathbf{s}) p_{h}}=\frac{\sum_{\mathbf{s} \in \mathbf{S}} \pi(\mathbf{s}) \gamma^{h}(\mathbf{s}) e(\mathbf{s})}{\sum_{\mathbf{s} \in \mathbf{S}} \pi(\mathbf{s}) e(\mathbf{s})} \\
& P_{\text {loss }}^{l}=\frac{\sum_{\mathbf{s} \in \mathbf{S}} \pi(\mathbf{s}) \gamma^{l}(\mathbf{s}) e(\mathbf{s}) p_{l}}{\sum_{\mathbf{s} \in \mathbf{S}} \pi(\mathbf{s}) e(\mathbf{s}) p_{l}}=\frac{\sum_{\mathbf{s} \in \mathbf{S}} \pi(\mathbf{s}) \gamma^{l}(\mathbf{s}) e(\mathbf{s})}{\sum_{\mathbf{s} \in \mathbf{S}} \pi(\mathbf{s}) e(\mathbf{s})}
\end{aligned}
$$


TABLE III

State Transitions From a Given State $\mathbf{s}=\left(s^{0}, s^{1}, \ldots, s^{L}, 0,0\right) \in \mathbf{S}$ FOR SOBS WITH QOS DIFFERENTIATION USING UNITY-OFFSET

\begin{tabular}{|l|l|l|}
\hline \multicolumn{3}{|c|}{ Current State: $\mathbf{s}=\left(s^{0}, s^{1}, \ldots, s^{L}, 0,0\right)$} \\
\hline Next State: $\mathbf{s}=$ & \multicolumn{1}{c|}{ Condition } & Probability \\
\hline$\left(s^{0}+s^{1}, s^{2}, \ldots, s^{L}, 0,0,0\right)$ & & $\alpha_{0}$ \\
\hline \hline$\left(s^{0}-1, s^{1}, \ldots, s^{L}+1,0,1\right)$ & $s^{0}>0$ & $\alpha_{1} p_{l}$ \\
\hline$\left(s^{0}, s^{1}, \ldots, s^{L}, 0,1\right)$ & $s^{0}=0$ & $\alpha_{1} p_{l}$ \\
\hline$\left(s^{0}-1, s^{1}, \ldots, s^{L}+1,0,2\right)$ & $s^{0}>0$ & $\alpha_{2} p_{l}$ \\
\hline$\left(s^{0}, s^{1}, \ldots, s^{L}, 0,2\right)$ & $s^{0}=0$ & $\alpha_{2} p_{l}$ \\
\hline \hline$\left(s^{0}, s^{1}-1, \ldots, s^{L}, 1,1\right)$ & $s^{1}>0$ & $\alpha_{1} p_{h}$ \\
\hline$\left(s^{0}-1, s^{1}, \ldots, s^{L}, 1,1\right)$ & $s^{1}=0, s^{0}>0$ & $\alpha_{1} p_{h}$ \\
\hline$\left(s^{0}, s^{1}, \ldots, s^{L}, 0,1\right)$ & $s^{1}=0, s^{0}=0$ & $\alpha_{1} p_{h}$ \\
\hline$\left(s^{0}, s^{1}-1, \ldots, s^{L}, 1,2\right)$ & $s^{1}>0$ & $\alpha_{2} p_{h}$ \\
\hline$\left(s^{0}-1, s^{1}, \ldots, s^{L}, 1,2\right)$ & $s^{1}=0, s^{0}>0$ & $\alpha_{2} p_{h}$ \\
\hline$\left(s^{0}, s^{1}, \ldots, s^{L}, 0,2\right)$ & $s^{1}=0, s^{0}=0$ & $\alpha_{2} p_{h}$ \\
\hline
\end{tabular}

TABLE IV

State Transitions From a Given State $\mathbf{s}=\left(s^{0}, s^{1}, \ldots, s^{L}, s^{L+1}, 1\right) \in \mathbf{S}$ FOR SOBS WITH QOS DIFFERENTIATION USING UNITY-OFFSET

\begin{tabular}{|c|c|c|}
\hline \multicolumn{3}{|c|}{ Current State: $\mathbf{s}=\left(s^{0}, s^{1}, \ldots, s^{L}, s^{L+1}, 1\right)$} \\
\hline Next State: $s=$ & Condition & Probability \\
\hline$\left(s^{0}+s^{1}, s^{2}, \ldots, s^{L}, s^{L+1}, 0,0\right)$ & & $\beta_{1}$ \\
\hline$\left(s^{0}-1, s^{1}, \ldots, s^{L}+1, s^{L+1}, 1\right)$ & $s^{0}>0$ & $\left(1-\beta_{1}\right) p_{l}$ \\
\hline$\left(s^{0}, s^{1}, \ldots, s^{L}, s^{L+1}, 1\right)$ & $s^{0}=0$ & $\left(1-\beta_{1}\right) p_{l}$ \\
\hline$\left(s^{0}, s^{1}-1, \ldots, s^{L}, s^{L+1}+1,1\right)$ & $s^{1}>0$ & $\left(1-\beta_{1}\right) p_{h}$ \\
\hline$\left(s^{0}-1, s^{1}, \ldots, s^{L}, s^{L+1}+1,1\right)$ & $s^{1}=0, s^{0}>0$ & $\left(1-\beta_{1}\right) p_{h}$ \\
\hline$\left.s^{L}, s^{L+1}, 1\right)$ & $s^{1}=0, s^{0}=0$ & $\left(1-\beta_{1}\right) p_{h}$ \\
\hline
\end{tabular}

TABLE V

State Transitions From a Given State $\mathbf{s}=\left(s^{0}, s^{1}, \ldots, s^{L}, s^{L+1}, 2\right) \in \mathbf{S}$ FOR SOBS WITH QOS DIFFERENTIATION USING UNITY-OFFSET

\begin{tabular}{|l|l|l|}
\hline \multicolumn{3}{|c|}{ Current State: $\mathbf{s}=\left(s^{0}, s^{1}, \ldots, s^{L}, s^{L+1}, 2\right)$} \\
\hline \multicolumn{1}{|c|}{ Next State: $\mathbf{s}=$} & \multicolumn{1}{c|}{ Condition } & Probability \\
\hline$\left(s^{0}-1, s^{1}, \ldots, s^{L}+1, s^{L+1}, 2\right)$ & $s^{0}>0$ & $\left(1-\beta_{2}\right) p_{l}$ \\
\hline$\left(s^{0}, s^{1}, \ldots, s^{L}, s^{L+1}, 2\right)$ & $s^{0}=0$ & $\left(1-\beta_{2}\right) p_{l}$ \\
\hline$\left(s^{0}-1, s^{1}, \ldots, s^{L}+1, s^{L+1}, 1\right)$ & $s^{0}>0$ & $\beta_{2} p_{l}$ \\
\hline$\left(s^{0}, s^{1}, \ldots, s^{L}, s^{L+1}, 1\right)$ & $s^{0}=0$ & $\beta_{2} p_{l}$ \\
\hline \hline$\left(s^{0}, s^{1}-1, \ldots, s^{L}, s^{L+1}+1,2\right)$ & $s^{1}>0$ & $\left(1-\beta_{2}\right) p_{h}$ \\
\hline$\left(s^{0}-1, s^{1}, \ldots, s^{L}, s^{L+1}+1,2\right)$ & $s^{1}=0, s^{0}>0$ & $\left(1-\beta_{2}\right) p_{h}$ \\
\hline$\left(s^{0}, s^{1}, \ldots, s^{L}, s^{L+1}, 2\right)$ & $s^{1}=0, s^{0}=0$ & $\left(1-\beta_{2}\right) p_{h}$ \\
\hline$\left(s^{0}, s^{1}-1, \ldots, s^{L}, s^{L+1}+1,1\right)$ & $s^{1}>0$ & $\beta_{2} p_{h}$ \\
\hline$\left(s^{0}-1, s^{1}, \ldots, s^{L}, s^{L+1}+1,1\right)$ & $s^{1}=0, s^{0}>0$ & $\beta_{2} p_{h}$ \\
\hline$\left(s^{0}, s^{1}, \ldots, s^{L}, s^{L+1}, 1\right)$ & $s^{1}=0, s^{0}=0$ & $\beta_{2} p_{h}$ \\
\hline
\end{tabular}

\section{NUMERICAL RESULTS}

In this section, we evaluate the performances of the three QoS mechanisms presented in Sections III, IV, and V. The results are obtained assuming that BCP arrival rates of HP and LP bursts

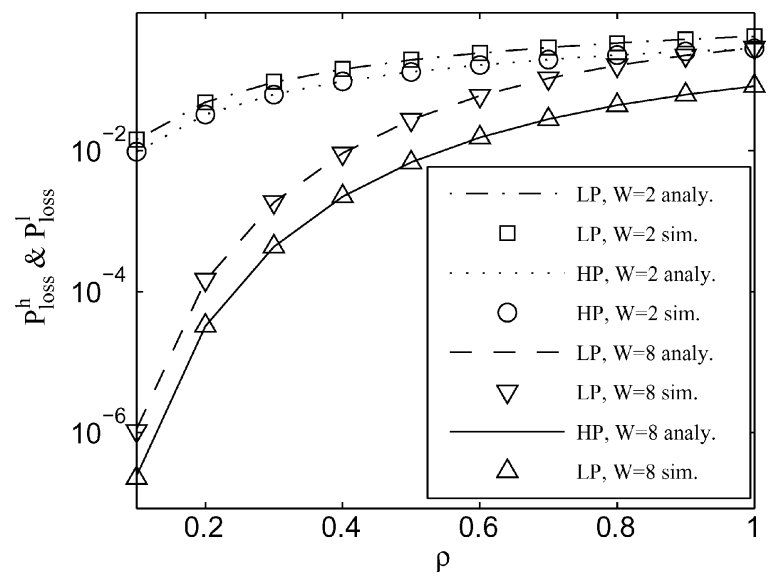

Fig. 6. Analytical and simulation results for burst loss probabilities of priority scheduling based QoS with $L=3$ and $W=2$ and 8 .

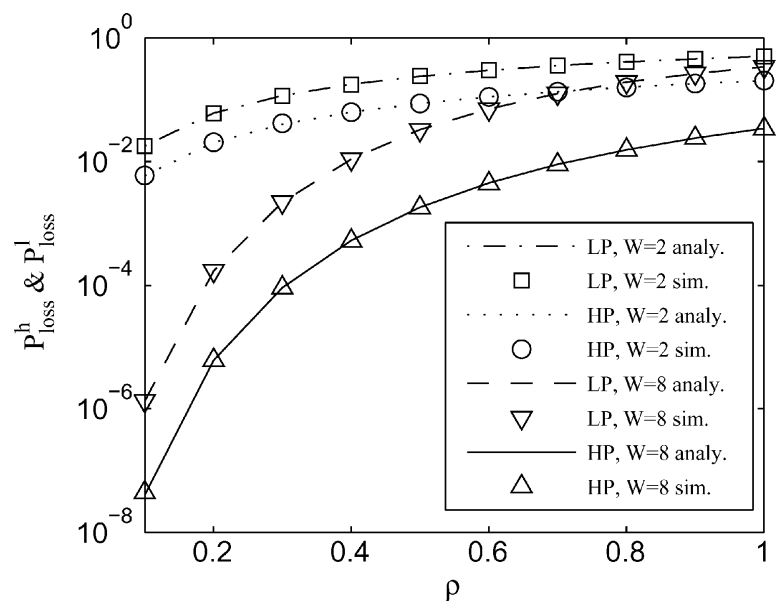

Fig. 7. Analytical and simulation results for burst loss probabilities of hybrid priority scheduling and unity-offset-based QoS with $L=3$ and $W=2$ and 8 .

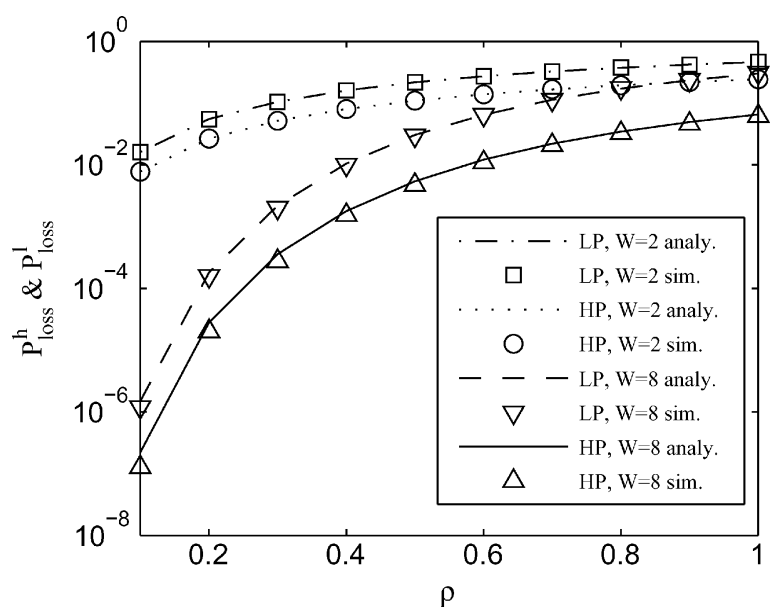

Fig. 8. Analytical and simulation results for burst loss probabilities of unityoffset-based QoS with $L=3$ and $W=2$ and 8 .

are equal unless stated otherwise, i.e., $p_{l}=p_{h}=1 / 2$. In Figs. 6 , 7 , and 8, analytical and simulation results for the systems analyzed in Sections III, IV, and V, respectively, are presented. In these experiments, the ratio of the burst length to the slot length, $L$, is taken as 3 . For the analysis of priority scheduling 


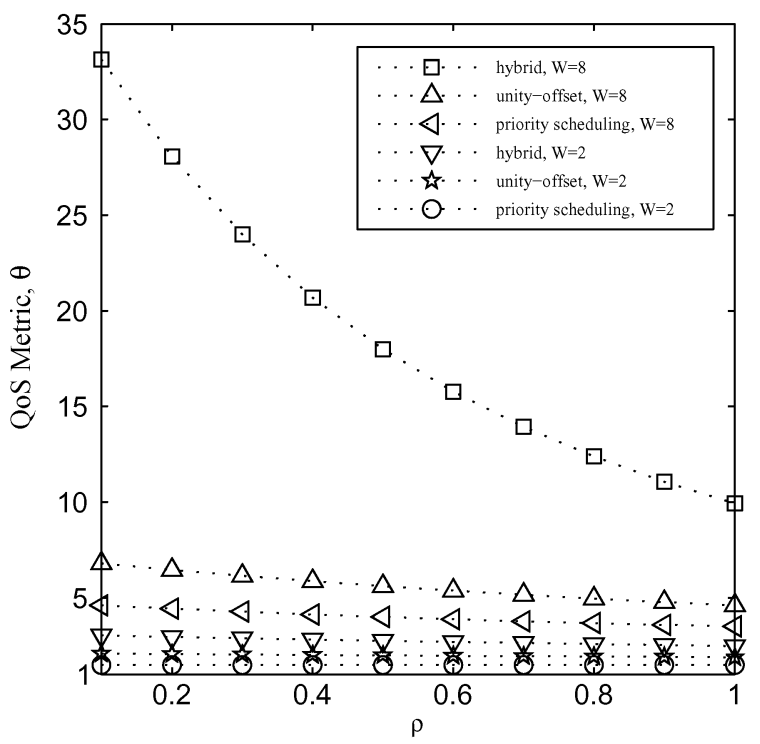

(a)

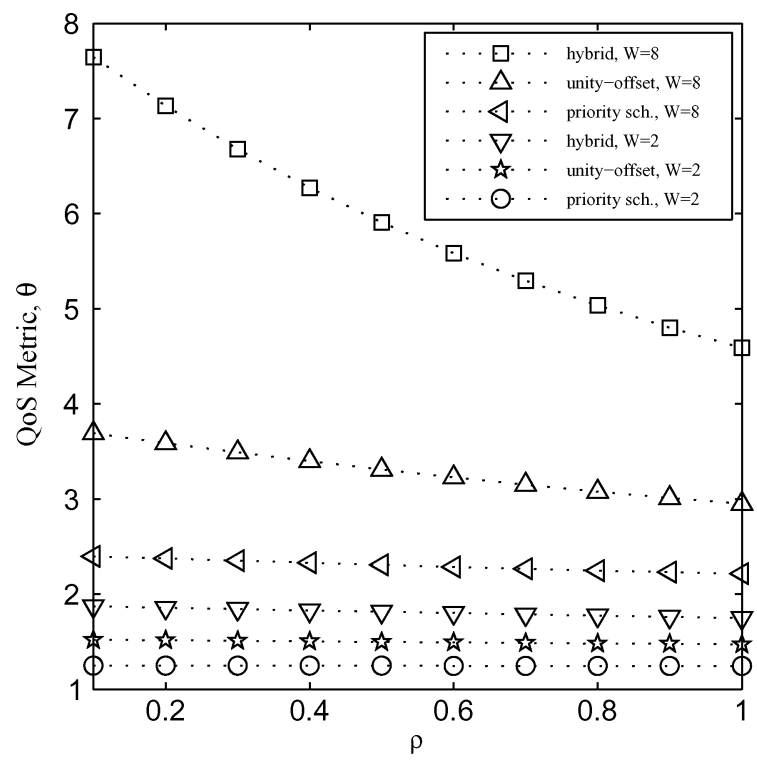

(b)

Fig. 9. QoS metric $\Theta$ of the three QoS schemes as a function of $\rho, W$ and $L$. (a) $L=3$. (b) $L=5$.

and hybrid priority scheduling with unity-offset-based differentiation methods, no further assumptions or approximations are made on top of the presumed models. Therefore, the simulation results for these two cases perfectly match with the results obtained by solving the analytical models. Meanwhile, for the unity-offset-based differentiation, we approximate the Poisson arrival process with a 2-phase acyclic D-PH type distribution as described in Section V. Although the first two moments of this arrival process and the PH type distribution are exactly matched, it is evident from Fig. 8 that as the number of wavelengths $W$ increases, under light traffic loads, $\rho$, mismatch in higher order moments yields a slightly pessimistic estimate for the burst loss probability especially for the high priority class.

In Fig. 9(a) and (b), performances of the three QoS mechanisms are compared for $L=3,5$ and $W=2,8$ as a function of $\rho$ by using the analytical models. We define a QoS metric $\Theta$ as the ratio of the loss probabilities for LP and HP bursts:

$$
\Theta=\frac{P_{\text {loss }}^{l}}{P_{\text {loss }}^{h}}
$$

The parameter $\Theta$ proves to grow with decreasing load $\rho$, decreasing burst length $L$, and increasing total number of wavelengths $W$. Among the three proposed QoS schemes, the hybrid mechanism outperforms the others as far as QoS differentiation metric $\Theta$ is concerned. These figures also reveal that HP bursts benefit from the unity QoS offset slightly more than the priority scheduling.

In Fig. 10, we use the analytical model presented in Section IV for the hybrid QoS scheme. For constant traffic load $\rho$, and burst length $L$, the effects of the proportion of the traffic belonging to the LP class on the loss probabilities are illustrated. The dashed lines in this figure shows the overall burst loss probability $P_{\text {loss }}$ of the core node, which is given by

$$
P_{\mathrm{loss}}=P_{\mathrm{loss}}^{l} p_{l}+P_{\mathrm{loss}}^{h}\left(1-p_{l}\right) \text {. }
$$

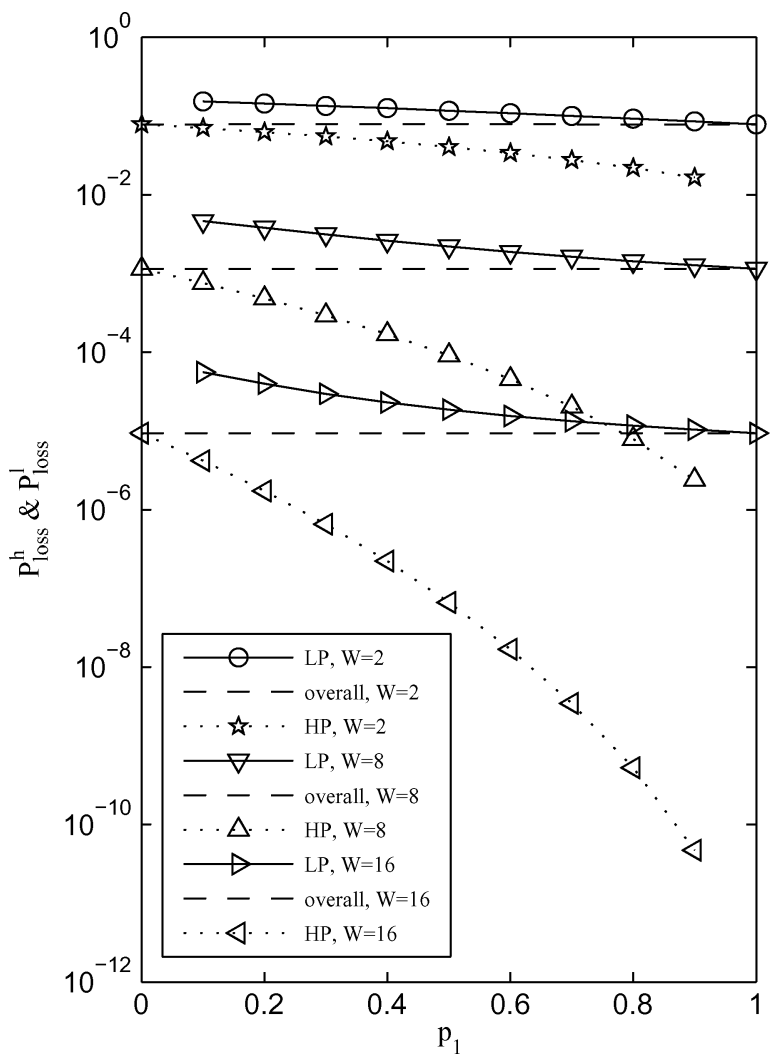

Fig. 10. Burst loss probabilities of hybrid priority scheduling with unity-offsetbased QoS with $L=3$ and $\rho=0.3$.

It is observed that $P_{\text {loss }}$ remains constant for varying $p_{l}$ and intersects the high priority and low priority curves at $p_{l}=0$ and $p_{l}=1$ for all values of $W$. This behavior is expected since when only a single class arrives at the optical node, QoS advantages and disadvantages disappear. It is evident from Fig. 10 that, as $p_{l}$ gets close to $1, \Theta$ rapidly increases since HP bursts become 


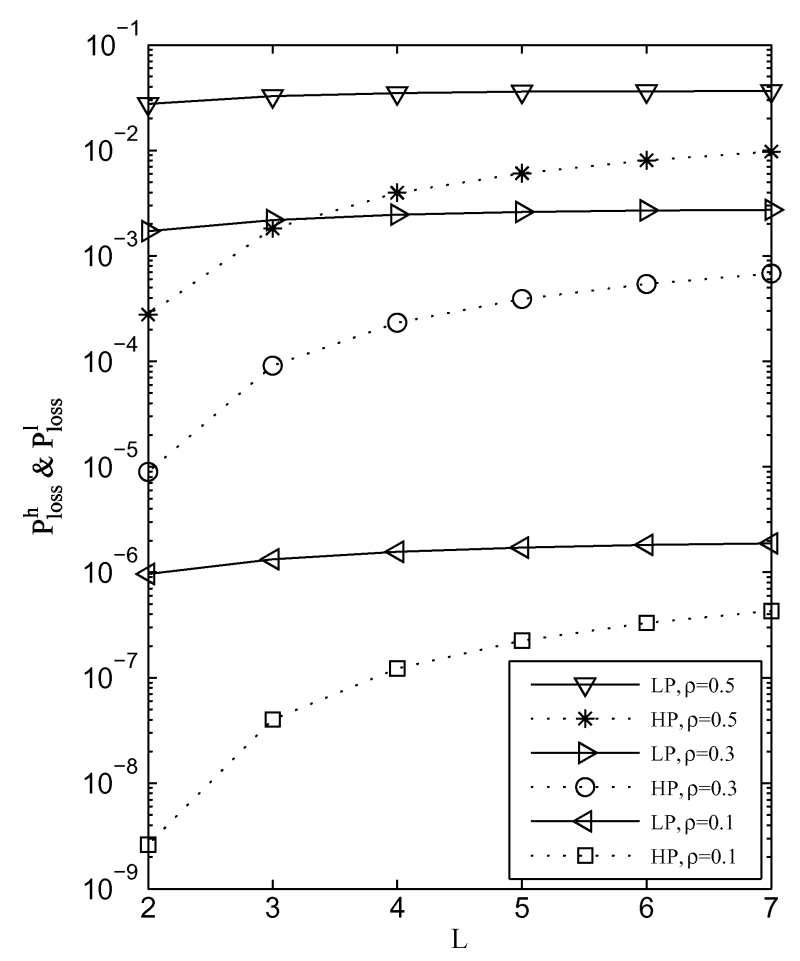

Fig. 11. Burst loss probabilities of hybrid priority scheduling with unity-offsetbased QoS for $W=8$.

less likely to block each other. On the contrary, LP bursts begin to collide with each other more frequently as $p_{l}$ increases.

In Fig. 11, the performance of the hybrid QoS scheme is evaluated as a function of the burst length to slot length ratio $L$ by using the analytical model presented in Section IV. In this figure, curvatures of the burst loss probability curves become steeper near $L=2$. At that point, full isolation is achieved between the HP and LP classes, and $P_{\text {loss }}^{h}$ becomes completely insensitive to the variations in the offered LP traffic load. When $L=2$, arriving LP bursts try to make reservations for two data slots, which reduce to a single data slot at the next coming slot boundary. By the advantage of their QoS offset, HP bursts do not differentiate between the wavelengths that are already available or that will be available in one data slot later as far as the available number of wavelengths are concerned. By the priority scheduling scheme, they also have complete priority over LP bursts in reserving available channels. Being unaffected by LP arrivals in these two cases, HP bursts are said to be fully isolated from the LP class when $L=2$. This isolation gradually decreases as $L$ increases and in the limit as $L \rightarrow \infty$, the advantage stemming from both the unity QoS offset and priority scheduling becomes negligible. In Fig. 11, $P_{\text {loss }}^{l}$ appears to be nearly independent from $L$, which shows that the decreasing level of prioritization and convergence to the asynchronous OBS regime cancel out each other as $L$ increases.

In Fig. 12, by using the analytical model for the hybrid QoS mechanism, it is shown that the burst loss probabilities drop significantly for increasing $W$ under low load conditions. This behavior is in accordance with $M / M / c / c$ loss systems, where loss probabilities decrease as the degree of statistical multiplexing increases with increasing number of servers, $c$.

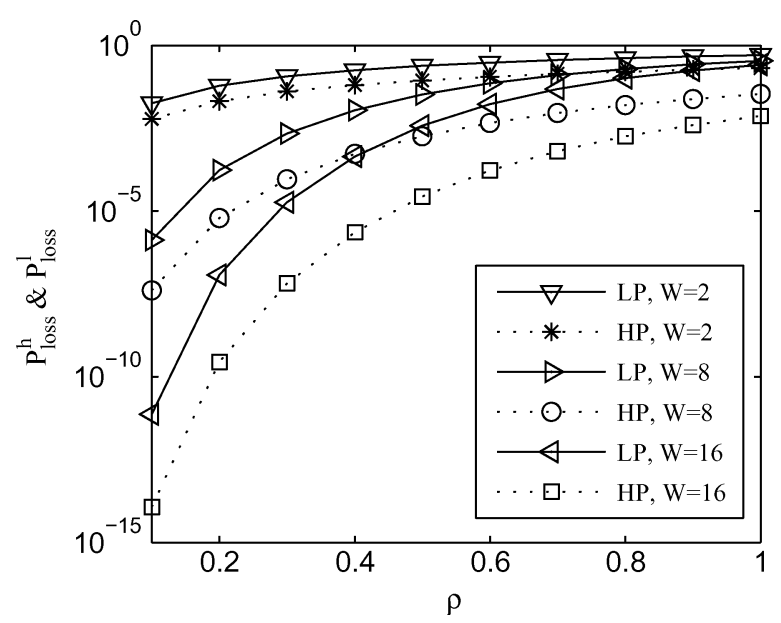

Fig. 12. Burst loss probabilities of hybrid priority scheduling with unity-offsetbased QoS scheme for $L=3$.

The last set of results are obtained for non-unity offset-based QoS mechanism. In Fig. 13(a) and (b), simulation results for the offset-based and hybrid QoS schemes with non-unity offsets are depicted. When the QoS offset $M$ is increased, it is observed that $P_{\text {loss }}^{h}$ decreases approximately linearly in the logarithmic scale. When $M$ reaches a threshold, $P_{\text {loss }}^{h}$ saturates since strict isolation between HP and LP bursts is attained. Once the strict isolation regime is reached, $P_{\text {loss }}^{l}$ curves change their curvatures as well, because an LP burst can no longer block an HP burst.

Generalizing the unity-offset-based QoS analysis in Sections IV and V to non-unity offsets requires a substantial increase in size of the state space of the constructed Markov chains. On the other hand, $P_{\text {loss }}^{h}$ corresponding to some particular values of $M$ can be computed without extensive computations as follows:

- For the offset-based QoS scheme:

- When $M=0$, HP and LP classes unify, i.e., $P_{\text {loss }}^{h}=$ $P_{\text {loss }}^{l}$, hence the Markov chain model, which was introduced in Section II for the best-effort SOBS node with a traffic load $\rho$, can be used to calculate this probability,

- For $M=1$, the Markov chain introduced in Section V can be used to compute $P_{\text {loss }}^{h}$ and $P_{\text {loss }}^{l}$,

- When $M \geq L$, HP bursts experience a network load consisting of only themselves. Thus, the same Markov chain but with a traffic load of $p_{h} \rho$ can be used for calculating $P_{\text {loss }}^{h}$.

- For the hybrid QoS scheme:

- When $M=0, \mathrm{HP}$ and LP classes experience only a priority scheduling based differentiation, hence the Markov chain introduced in Section III can be used to calculate $P_{\text {loss }}^{h}$ and $P_{\text {loss }}^{l}$,

- For $M=1$, the Markov chain introduced in Section IV can be directly used to compute $P_{\text {loss }}^{h}$ and $P_{\text {loss }}^{l}$,

- When $M \geq L-1$, HP bursts experience a network load consisting of only themselves. Thus, the Markov chain for best-effort SOBS with a traffic load of $p_{h} \rho$ can be used for calculating $P_{\text {loss }}^{h}$ (note that because of the preferential treatment of HP bursts among the bursts arriving in the same time slot, HP bursts do not need to 


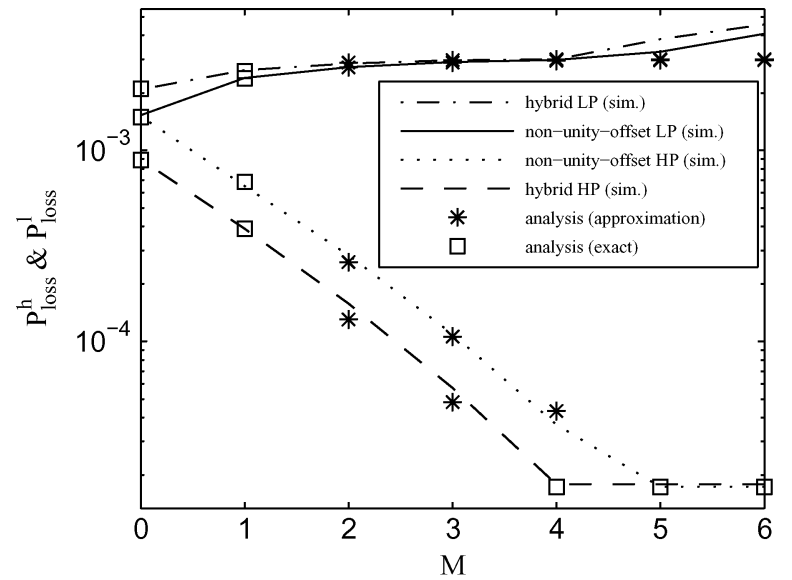

(a)

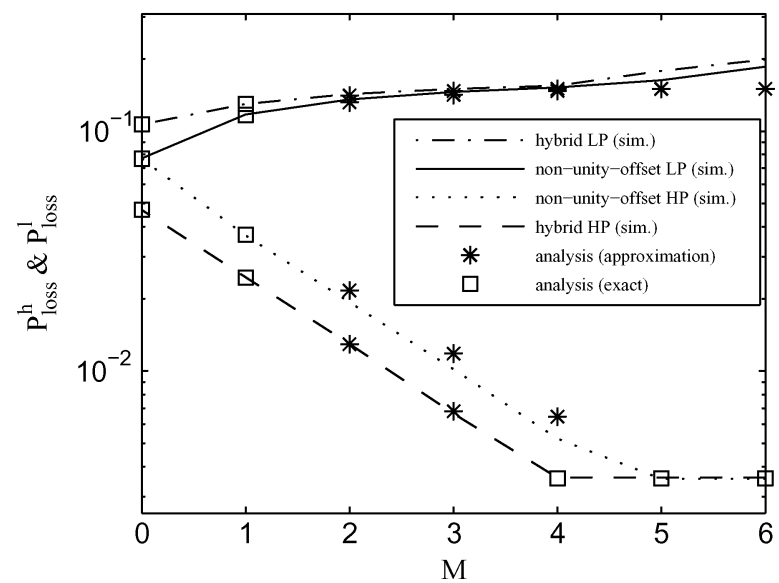

Fig. 13. Burst loss probability estimations of non-unity-offset-based QoS schemes for changing QoS offset length, $M$, with $L=5$ and $W=8$. (a) $\rho=0.3$. (b) $\rho=0.7$.

have $M=L$ in order to have complete isolation from LP bursts.)

$P_{\text {loss }}^{h}$ and $P_{\text {loss }}^{l}$ values obtained by using the exact analysis for $M=0,1$, and $P_{\text {loss }}^{h}$ obtained for $M \geq L-1$, as outlined above, are marked with " $\square$ " in Fig. 13(a) and (b) for two different traffic loads, $\rho=0.3$ and $\rho=0.7$. Once these points are computed, we use a least squares solution to compute $P_{\text {loss }}^{h}$ for the intermediate points. The points obtained through this approximation are marked with " $*$ " in the figure. After computing $P_{\text {loss }}^{h}$, we next compute $P_{\text {loss }}^{l}$ as follows. Assuming that both systems are work conserving, $P_{\text {loss }}^{l}$ for non-unity offset-based and hybrid QoS schemes can be calculated from the overall loss probabilities, $P_{\text {loss }}$, of a best-effort SOBS node and an SOBS node with priority scheduling, respectively, with load $\rho$, and by using the relation

$$
P_{\text {loss }}=\left(1-p_{l}\right) P_{\text {loss }}^{h}+p_{l} P_{\text {loss }}^{l} \text {. }
$$

The $P_{\text {loss }}^{l}$ values obtained from the above approximation procedure are also marked with "*” in Fig. 13(a) and (b). As seen in the figure, the work conservation assumption is not valid especially for $M>L$. Nevertheless, using this procedure, $P_{\text {loss }}^{h}$ and $P_{\text {loss }}^{l}$ can be computed with an acceptable accuracy for this particular case, without requiring significant amount of computations.

\section{CONCLUSION}

In this paper, we developed a Markov chain based framework for performance evaluation of SOBS by which we studied the burst loss probabilities of both a best-effort and a prioritized SOBS core node. We use this framework in order to compute the burst loss probabilities of these two kinds of SOBS nodes for varying traffic load, $\rho$, number of wavelengths, $W$, and burst length $L$. We validate the accuracy of our framework against simulations.

Results show that as $L$ increases, SOBS asymptotically converges to asynchronous OBS in terms of burst loss probability and this convergence is relatively faster for high loss rates where $\rho$ is high and $W$ is low. We also show that SOBS may provide a similar performance to the traditional OBS but with fewer wavelengths and under relatively heavy traffic conditions, increasing the chance of burst switching paradigm to be appraised as the next generation Internet architecture.

Among the QoS schemes analyzed in this paper, hybrid priority scheduling with offset-based differentiation yields the best isolation between the QoS classes and offset-based differentiation ranks the second leaving priority scheduling the last. As $W$ increases and $L$ decreases, a higher isolation level is achieved. By decreasing the proportion of the traffic consisting of high priority bursts, even higher degree in isolation can be reached.

We believe that the proposed framework presented in this paper can be further extended to analyze some other variations in an SOBS network which are listed as follows.

1) In this paper, BCP arrivals within a slot are assumed to be Poisson distributed. However, other distributions may be of interest. Proposed Markov chains, other than the one for the unity-offset differentiation, use only the PMF, CDF and the CCDF functions of a given arrival process. Hence they offer full flexibility in changing the $\mathrm{BCP}$ arrival distribution without sacrificing from precision. However, in the analysis of the unity-offset differentiation, the Poisson distribution is approximated by a D-PH type distribution. Although moment matching for another distribution can be done according to the formulation given in Appendix I, results would need validation.

2) Fixed length assumption for bursts can be generalized to variable length bursts with a certain probability distribution.

3) The case of non-unity QoS offsets, $M>1$, can be analyzed using improved computational techniques for large but sparse Markov chain state transition matrices.

4) The framework can be extended to the analysis of networks with more than two priority classes.

\section{APPENDIX I \\ MOMENT MATCHING FOR $\lambda$}

Let $\lambda=\lambda_{h}+\lambda_{l}$. be the total BCP arrival rate to the optical node and $X$ be the random variable denoting the number of 
arrivals generated by the D-PH type distribution given in Fig. 5 . Then, the probability-generating function of $X$ is written as

$$
\begin{aligned}
G_{X}(z)= & E\left[z^{X}\right] \\
= & \alpha_{0}+\alpha_{1} \frac{\beta_{1} z}{1-\left(1-\beta_{1}\right) z} \\
& +\alpha_{2} \frac{\beta_{2} z}{1-\left(1-\beta_{2}\right) z} \frac{\beta_{1} z}{1-\left(1-\beta_{1}\right) z} .
\end{aligned}
$$

The first three factorial moments of $X$ are defined as follows:

$$
\begin{aligned}
f_{1} & :=\left.\frac{d}{d z} G_{X}(z)\right|_{z=1}=E[X] \\
f_{2} & :=\left.\frac{d^{2}}{d z^{2}} G_{X}(z)\right|_{z=1}=E[X(X-1)] \\
f_{3} & :=\left.\frac{d^{3}}{d z^{3}} G_{X}(z)\right|_{z=1}=E[X(X-1)(X-2)] .
\end{aligned}
$$

Let $Z$ be the Poisson distributed random variable with rate $\lambda$. Then, the following equalities should hold for a perfect moment matching between $Z$ and $X$ :

$$
\begin{aligned}
f_{1} & =E[Z]=\lambda \\
f_{2} & =E\left[Z^{2}-Z\right]=\left(\lambda^{2}+\lambda\right)-\lambda=\lambda^{2} \\
f_{3} & =E\left[Z^{3}-3 Z^{2}+2 Z\right] \\
& =\left(\lambda^{3}+3 \lambda^{2}+\lambda\right)-3\left(\lambda^{2}+\lambda\right)+2 \lambda \\
& =\lambda^{3} .
\end{aligned}
$$

However, simultaneously solving (3), (4), and (5) is non-feasible for a broad range of $\lambda$. Even the second moment formulated by (4) requires $\lambda$ to be lower than a threshold which is found to be approximately 3.72 in Appendix II. Hence, we converted this moment matching problem into a constrained optimization problem for this analysis as follows:

$$
\min _{\alpha_{0}, \alpha_{1}, \alpha_{2}, \beta_{1}, \beta_{2}}\left|f_{3}-\lambda^{3}\right|
$$

such that

$$
\begin{aligned}
f_{1} & =\lambda \\
f_{2} & =\lambda^{2} \\
\alpha_{0} & =q_{(0)} \\
0 & \leq \alpha_{1}, \alpha_{2}, \beta_{1}, \beta_{2} \leq 1 \\
\alpha_{0}+\alpha_{1}+\alpha_{2} & =1 .
\end{aligned}
$$

Solution to (6) and (7) in the feasible range of $\lambda$ gives the parameters $\alpha_{0}, \alpha_{1}, \alpha_{2}, \beta_{1}$, and $\beta_{2}$ of the D-PH type distribution shown in Fig. 5.

\section{APPENDIX II}

\section{UPPER BOUND CALCULATION FOR $\lambda$}

Here we calculate the upper bound for $\lambda$ below which (3) and (4) are guaranteed to be satisfied. They are explicitly solved as follows:

$$
f_{1}=\frac{\alpha_{1} \beta_{2}+\alpha_{2} \beta_{1}+\alpha_{2} \beta_{2}}{\beta_{1} \beta_{2}}=\lambda
$$

$$
\begin{aligned}
f_{2}= & \frac{-2}{\beta_{1}^{2} \beta_{2}^{2}}\left(\alpha_{1} \beta_{1} \beta_{2}^{2}-\alpha_{1} \beta_{2}^{2}-\alpha_{2} \beta_{1} \beta_{2}\right. \\
& \left.\quad+\alpha_{2} \beta_{1}^{2} \beta_{2}+\alpha_{2} \beta_{1} \beta_{2}^{2}-\alpha_{2} \beta_{1}^{2}-\alpha_{2} \beta_{2}^{2}\right) \\
= & \lambda^{2} \quad
\end{aligned}
$$

where $\alpha_{0}=e^{-\lambda}$ and $\alpha_{1}=1-\alpha_{0}-\alpha_{2}$ according to (7). Eq. (8) can be solved for $\alpha_{2}$ after replacing $\alpha_{1}$ by $1-e^{-\lambda}-\alpha_{2}$ as follows:

$$
\alpha_{2}=\lambda \beta_{2}-\frac{\beta_{2}}{\beta_{1}}\left(1-e^{-\lambda}\right) .
$$

Similarly (9) can be simplified after replacing $\alpha_{1}$ :

$$
f_{2}=\alpha_{2} \frac{2\left(\beta_{1}-\beta_{1} \beta_{2}+\beta_{2}\right)}{\beta_{1} \beta_{2}^{2}}+\left(1-e^{-\lambda}\right) \frac{-2\left(\beta_{1}-1\right)}{\beta_{1}^{2}}=\lambda^{2} .
$$

Substituting $\alpha_{2}$ found in (10) into (11), we end up with the following equality:

$$
\lambda^{2}-\lambda \frac{2\left(\beta_{1}-\beta_{1} \beta_{2}+\beta_{2}\right)}{\beta_{1} \beta_{2}}+\left(1-e^{-\lambda}\right) \frac{2}{\beta_{1} \beta_{2}}=0 .
$$

Eq. (12) should be solved for maximum $\lambda$ for which the following inequality inherited from (10) is satisfied:

$$
0 \leq \alpha_{2}=\lambda \beta_{2}-\frac{\beta_{2}}{\beta_{1}}\left(1-e^{-\lambda}\right)<1-e^{-\lambda} .
$$

The computations of the maximum value of $\lambda$ for which (12) can be solved such that (13) is satisfied do not have a simple closed form solution. By making use of numerical methods, an upper bound on $\lambda$ for which the first two moments of the D-PH type distribution matches with the Poisson distribution is obtained to be 3.72358161 for a precision of $10^{-8}$.

\section{REFERENCES}

[1] C. Qiao and M. Yoo, "Optical burst switching (OBS)—A new paradigm for an optical Internet," J. High Speed Netw., vol. 8, no. 1, pp. 69-84, 1999.

[2] S. Verma, H. Chaskar, and R. Ravikanth, "Optical burst switching: A viable solution for terabit IP backbone," IEEE Network Mag., vol. 14, no. 6 , pp. $48-53,2000$.

[3] J. Finochietto, J. Aracil, A. Ferreiro, J. P. Fernandez-Palacios Gimenez, and O. Gonzalez de Dios, "Migration strategies toward all optical metropolitan access rings," J. Lightw. Technol., vol. 25, no. 8, pp. 1918-1930, Aug. 2007.

[4] J. Berthold, A. A. M. Saleh, L. Blair, and J. M. Simmons, "Optical networking: Past, present, and future," J. Lightw. Technol., vol. 26, no. 9, pp. 1104-1118, 2008.

[5] "Total cost of ownership analysis of Matisse networks EtherBurst optical switch," Network Strategy Partners, LLC, White paper, 22 pp., Apr. 2007 [Online]. Available: http://www.nspllc.com/

[6] Matisse Networks. [Online]. Available: http://www.matissenetworks. com/

[7] Y. Chen, C. Qiao, and X. Yu, "Optical burst switching: A new area in optical networking research," IEEE Network Mag., vol. 18, no. 3, pp. 16-23, 2004.

[8] M. Yoo and C. Qiao, "Just-enough-time (JET): A high speed protocol for bursty traffic in optical networks," presented at the IEEE/LEOS Technologies for a Global Information Infrastructure Conf., Stockholm, Sweden, 1997.

[9] K. Lee and V. Li, "A wavelength convertible optical network," $J$. Lightw. Technol., vol. 11, pp. 962-970, 1993.

[10] R. A. Barry and P. Humblet, "Models of blocking probability in alloptical networks with and without wavelength changers," IEEE J. Sel. Areas Commun., vol. 14, pp. 858-867, 1996.

[11] C. Gauger, "Optimized combination of converter pools and FDL buffers for contention resolution in optical burst switching," Photon. Netw. Commun., vol. 8, no. 2, pp. 139-148, 2004. 
[12] A. Zalesky, H. L. Vu, Z. Rosberg, E. W. M. Wong, and M. Zukerman, "Stabilizing deflection routing in optical burst switched networks," IEEE J. Sel. Areas Commun., vol. 25, no. 6, pp. 3-19, 2007.

[13] N. Akar, E. Karasan, and K. Dogan, "Wavelength converter sharing in asynchronous optical packet/burst switching: An exact blocking analysis for Markovian arrivals," IEEE J. Sel. Areas Commun., vol. 24, no. 12 (Part supplement), pp. 69-80, 2006

[14] S. Yao, S. J. B. Yoo, B. Mukherjee, and S. Dixit, "All-optical packet switching for metropolitan area networks: Opportunities and challenges," IEEE Commun. Mag., vol. 39, no. 3, pp. 142-148, 2001.

[15] F. Farahmand, V. Vokkarane, and J. Jue, "Practical priority contention resolution for slotted optical burst switching networks," presented at the 1st Int. Workshop Optical Burst Switching (WOBS), Dallas, TX, 2003.

[16] A. Rugsachart, "Time synchronized optical burst switching," Ph.D. dissertation, University of Pittsburgh, Pittsburgh, PA, Aug. 2007.

[17] Z. Zhang, L. Liu, and Y. Yang, "Slotted optical burst switching (SOBS) networks," Comput. Commun., vol. 30, no. 8, pp. 3471-3479, 2007.

[18] J. Li, C. Qiao, J. Xu, and D. Xu, "Maximizing throughput for optical burst switching networks," IEEE/ACM Trans. Netw., vol. 15, no. 5, pp. 1163-1176, Oct. 2007.

[19] M. Yoo, C. Qiao, and S. Dixit, "QoS performance of optical burst switching in IP-over-WDM networks," IEEE J. Sel. Areas Commun., vol. 18, pp. 2062-2071, Oct. 2000.

[20] Z. Rosberg, H. L. Vu, M. Zukerman, and J. White, "Performance analyses of optical burst-switching networks," IEEE J. Sel. Areas Commun., vol. 21, no. 7, pp. 1187-1197, Sep. 2003.

[21] A. Zalesky, H. L. Vu, Z. Rosberg, E. W. M. Wong, and M. Zukerman, "OBS contention resolution performance," Perform. Eval., vol. 64, no. 4, pp. 357-373, 2007.

[22] S. Asmussen and J. R. Moller, "Calculation of the steady state waiting time distribution in GI/PH/c and MAP/PH/c queues," Queueing Syst., vol. 37, no. 1/3, pp. 9-29, 2001.

[23] M. Telek and A. Heindl, "Matching moments for acyclic discrete and continous phase-type distributions of second order," Int. J. Simulation Syst., Sci. Technol., vol. 3, no. 3-4, pp. 47-57, Dec. 2002.

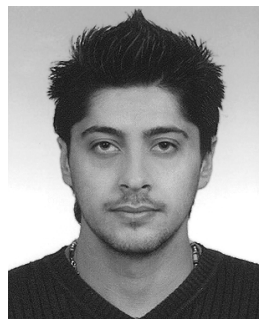

Onur Ozturk received the B.S. degree from Middle East Technical University, Ankara, Turkey, and the M.S. degree from Bilkent University, Ankara, Turkey, both in electrical and electronics engineering, in 2005 and 2008, respectively. $\mathrm{He}$ is currently working towards the Ph.D. degree at Bilkent University.

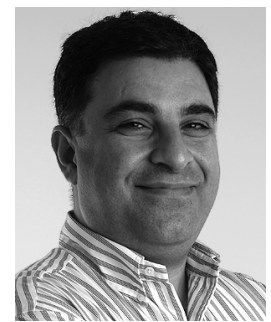

Ezhan Karasan (S'88-M'95) received the B.S degree from Middle East Technical University, Ankara, Turkey, the M.S. degree from Bilkent University, Ankara, Turkey, and the Ph.D. degree from Rutgers University, Piscataway, NJ, all in electrical engineering, in 1987, 1990, and 1995, respectively.

During 1995-1996, he was a Postdoctorate Researcher at Bell Labs, Holmdel, NJ. From 1996 to 1998, he was a Senior Technical Staff Member in the Lightwave Networks Research Department at AT\&T Labs-Research, Red Bank, NJ. He has been with the Department of Electrical and Electronics Engineering at Bilkent University since 1998, where he is currently an Associate Professor. He has been participating in the FP6-IST Network of Excellence (NoE) e-Photon/ONe+ and FP7-IST NoE BONE projects. His current research interests are in the application of optimization and performance analysis tools for the design, engineering and analysis of optical networks and wireless ad hoc/mesh/sensor networks.

Dr. Karasan is a member of the Editorial Board of Optical Switching and Networking Journal. He was the recipient of the 2004 Young Scientist Award from the Turkish Scientific and Technical Research Council (TUBITAK), 2005 Young Scientist Award from the Mustafa Parlar Foundation and the Career Grant from TUBITAK in 2004. He received a fellowship from the NATO Science Scholarship Program for overseas studies in 1991-1994.

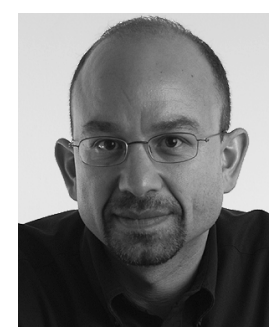

Nail Akar (M'94) received the B.S. degree from Middle East Technical University, Ankara, Turkey, in 1987, and the M.S. and Ph.D. degrees from Bilkent University, Ankara, Turkey, in 1989 and 1994, respectively, all in electrical and electronics engineering.

From 1994 to 1996, he was a Visiting Scholar and a Visiting Assistant Professor in the Computer Science Telecommunications program at the University of Missouri-Kansas City. In 1996, he joined the Technology Planning and Integration group at the Long Distance Division, Sprint, where he was a Senior Member of Technical Staff from 1999 to 2000. Since 2000, he has been a faculty member at Bilkent University, currently as an Associate Professor. His current research interests include performance analysis of computer and communication networks, queueing systems, traffic engineering, network control and resource allocation, and optical networking.

Dr. Akar actively participates in European Commission FP7 NoE projects BONE and WiMAGIC. 